\title{
Studying an effect of salt powder seeding used for precipitation enhancement from convective clouds
}

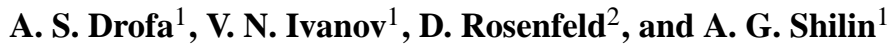 \\ ${ }^{1}$ Institute of Experimental Meteorology, Research and Production Association “Typhoon”, Obninsk, Russia \\ ${ }^{2}$ Institute of Earth Sciences, The Hebrew University of Jerusalem, Israel
}

Received: 10 February 2010 - Published in Atmos. Chem. Phys. Discuss.: 23 April 2010

Revised: 11 August 2010 - Accepted: 23 August 2010 - Published: 27 August 2010

\begin{abstract}
Experimental and theoretical studies of cloud microstructure modification with hygroscopic particles for obtaining additional precipitation amounts from convective clouds are performed. The experiment used salt powder with the particle sizes that gave the greatest effectiveness according to the simulations of Segal et al. (2004). The experiments were carried out in a cloud chamber at the conditions corresponding to the formation of convective clouds. The results have shown that the introduction of the salt powder before a cloud medium is formed in the chamber results in the formation on a "tail" of additional large drops. In this case seeding with the salt powder leads also to enlargement of the whole population of cloud drops and to a decrease of their total concentration as compared to a cloud medium that is formed on background aerosols. These results are the positive factors for stimulating coagulation processes in clouds and for subsequent formation of precipitation in them. An overseeding effect, which is characterized by increased droplet concentration and decreased droplet size, was not observed even at high salt powder concentrations.

The results of numerical simulations have shown that the transformation of cloud drop spectra induced by the introduction of the salt powder results in more intense coagulation processes in clouds as compared to the case of cloud modification with hygroscopic particles with relatively narrow particle size distributions, and for the distribution of the South African hygroscopic flares. The calculation results obtained with a one-dimensional model of a warm convective cloud demonstrated that the effect of salt powder on clouds (total amounts of additional precipitation) is significantly higher than the effect caused by the use of hygroscopic particles with narrow particle size distributions at comparable con-
\end{abstract}

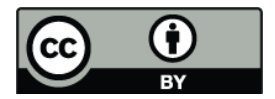

Correspondence to: D. Rosenfeld (daniel.rosenfeld@huji.ac.il) sumptions of seeding agents, or with respect to the hygroscopic flares. Here we show that seeding at rather low consumption rate of the salt powder initiates precipitation from otherwise non precipitating warm convective clouds, mainly by the effect of adding large cloud drops to the tail of the distribution.

\section{Introduction}

Operational hygroscopic cloud seeding aimed at rain enhancement has been conducted extensively in many countries, including India, USA, Saudi Arabia, China, Thailand and other countries. Hygroscopic seeding for rain enhancement in convective clouds is aimed at accelerating autoconversion, i.e., the conversion of cloud water to precipitation. This was reviewed extensively by Bruintjes (1999) and Silverman (2003). Three main conceptual models have guided the hygroscopic seeding experiments:

- The rain embryo particles: Seeding with ultra-giant CCN (size $>10 \mu \mathrm{m}$ ), which serve as embryos for raindrops.

- The tail effect: Seeding with giant CCN (diameter between 1 and $10 \mu \mathrm{m}$ ) adds drops to the large end tail of the DSD, and hence accelerates the further widening of the DSD and leads to formation of rain drops (Segal et al., 2004).

- The competition effect: Seeding with giant or large $\mathrm{CCN}$ (diameter near $1 \mu \mathrm{m}$ ), for greater competition for the vapor, decreasing peak super saturation at cloud base, and hence reducing cloud drop number concentrations and broadening the cloud drop size distribution (DSD). This causes larger drops that coalesce faster into rain drops (Cooper et al., 1997). 
The seeding practice with hygroscopic flares became fashionable after the reports of the apparent success of the South African (Mather et al., 1997) and Mexican (WMO, 2000; Bruintjes et al., 2001) experiments, and the simulations with theoretical support for the efficacy of the flares (Cooper et al., 1997). Mather et al. (1997) showed an isolated case where the DSD within a small volume of a cloud base was widened in the just-seeded concentrated seeded plume of the flares, and argued that this was caused by the competition effect. The operational practice has been to seed the updraft just below cloud base with one to two 1-kg hygroscopic flares that burn in about 4 minutes. This means a seeding rate of 0.25 to $0.5 \mathrm{~kg} / \mathrm{min}$ or, with an air speed of $70 \mathrm{~ms}^{-1}$, a rate of 0.015 $0.03 \mathrm{~kg} \mathrm{~km}^{-1}$ of seeding path. This practice prevails even though demonstration of its efficacy is lacking. Furthermore, Segal et al. (2004) calculated the particle size distribution of hygroscopic aerosols that would accelerate the conversion of cloud water to rain drops for the least amount of salt mass, and showed that this was quite different from the particle size distribution that is produced by burning hygroscopic flares. Rosenfeld et al. (2010) followed this up and manufactured a salt powder that matched the optimal particle size distribution as calculated by Segal et al. (2004), and tested it against hygroscopic flares in actual cloud seeding experiments. The results of Rosenfeld et al. (2010) support the simulations of Segal et al. (2004) and show that to produce a microphysical seeding effect on clouds that is detectable by cloud physics aircraft, a mass concentration greater by an order of magnitude than is presently practiced with hygroscopic flares has to be dispersed.

This paper presents the results of experimental and theoretical studies of cloud modification with the salt powder developed by Lahav and Rosenfeld (2005) and Rosenfeld et al. (2010) for obtaining additional precipitation amounts from convective clouds. Experiments were carried out in the cloud chamber of the Research and Production Association "Typhoon", Russia, under the conditions corresponding to the formation of convective clouds, as described in Sect. 2. The observed changes of the cloud microstructure at different seeding rates in the cloud chamber are given in Sect. 3. The conversion of cloud water into rain could not be fully documented even in this big cloud chamber due to sedimentation of the large cloud drops. The implication with respect to conversion of the cloud water to rainfall had to be assessed by a 1-dimensional cloud model, which replicated the observed initial spectra and extended the calculations through the formation of rain. This was done both for the salt powder and for other hygroscopic agents with narrow particle size distributions, as described in Sect. 4. The conclusions, given in Sect. 5, provide the particle size distribution and seeding rates that would result in the fastest conversion of cloud water to rainfall for a range of seeding rates of the hygroscopic seeding agents. It should be noted here that the fastest conversion of cloud water to rainfall does not always mean also the greatest amount of precipitation (Rosenfeld et al.,
2008). Establishing the seeding effects on precipitation requires randomized seeding experiments in the natural atmospheric conditions accompanied by detailed microphysical measurements and model simulations.

\section{The big cloud chamber and its usage for measurements in aerosol and cloud media}

Experimental studies of a salt powder seeding effect on a cloud medium were performed in the Big Cloud Chamber (BCC) of RPA "Typhoon". BCC is designed for modeling convective or stratified clouds and fogs of different origin under the conditions close to those in the real atmosphere. The chamber is a steel airtight cylinder of $18-\mathrm{m}$ height and $15 \mathrm{~m}$ diameter. The chamber walls of 6-mm thickness are externally heat-insulated. The total volume of the chamber is $3200 \mathrm{~m}^{3}$. A cloud medium is formed up to a height of $15 \mathrm{~m}$. The technological compartment separated by a metallic grating is located above. A detailed description of the BCC and the methods of cloud media formation in it are presented in (Romanov and Zhukov, 2000).

The process of convective cloud medium formation in the BCC is made through air expansion. For this, external air is pumped into the chamber. As a result the air pressure in it is increased to a certain level. The air pressure decrease is made by opening holes in the upper part of the chamber. The air discharge from the chamber through the holes of different cross sections makes it possible to regulate the rate of pressure decrease in the chamber, thus setting a certain velocity of air updraft in the atmosphere during the formation of a convective cloud. The program controlling pressure decreasing in the BCC makes it possible to realize a preset scenario of air mass updraft in the atmosphere with equivalent rates of $0.1-10 \mathrm{~ms}^{-1}$. Air temperature, pressure, humidity and the chamber wall temperatures are basic parameters for setting thermodynamic conditions in the BCC. These parameters are continuously measured during the experiments in the BCC. For setting the conditions of cloud medium formation thermodynamic relationships and the known heat exchange coefficients for the BCC are used (Romanov and Zhukov, 2000). The initial stage of cloud formation is simulated in the BCC at updraft velocities usually of $1-2 \mathrm{~ms}^{-1}$ for warm continental clouds. The cloud drop size distribution formed is determined by the physicochemical properties of condensation nuclei and the water vapor supersaturation in the chamber. The cloud drop concentration depends on maximum supersaturation. Water vapor supersaturation is not measured in the BCC, but in our experiments realized are cloud drop concentrations observed in continental convective clouds.

In the efforts described in this paper the pressure decrease in the BCC was made from the initial value of $1300 \mathrm{hPa}$ with the velocity of air mass updraft in the atmosphere of $1.2-1.3 \mathrm{~m} / \mathrm{s}$. Relative humidity of air in the chamber before the pressure drop is usually $90-96 \%$. The air temperature 


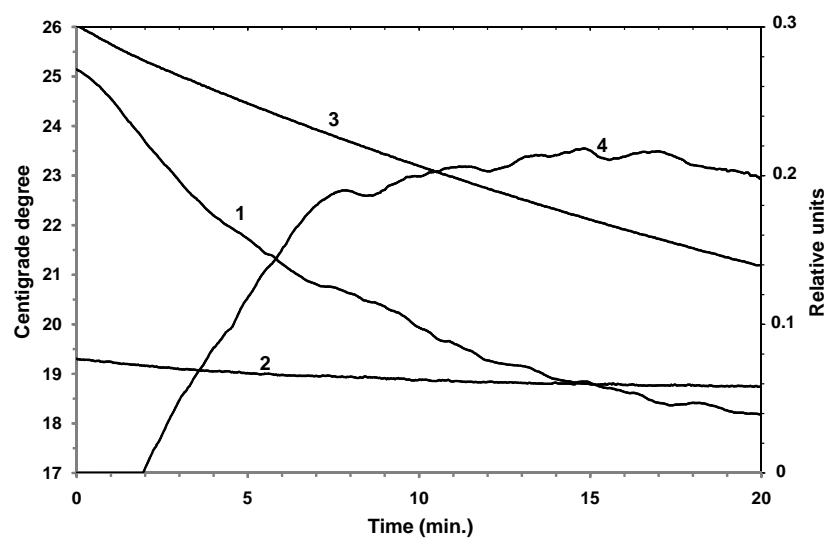

Fig. 1. Typical changes with time of the BCC parameters during convective cloud modeling. Air temperature and the wall chamber temperature variations (curves 1,2 , respectively, left scale), a relative excess of air pressure in the chamber $(\mathrm{Pc}-\mathrm{Po}) / \mathrm{Po}$ where $\mathrm{Pc}$ and Po are the chamber and outside pressures, respectively (curve 3, right scale), the coefficient of light attenuation in the cloud medium at the $0.63 \mu \mathrm{m}$ wavelength $\left(4, \mathrm{~m}^{-1}\right.$, right scale). The time count begins at the moment of the beginning of the pressure lowering.

is $22-25^{\circ} \mathrm{C}$. At such values of meteorological parameters a cloud medium in the $\mathrm{BCC}$ is formed in $2 \mathrm{~min}$ after the beginning of the pressure lowering (see Fig. 1). By this time the air humidity attains $100 \%$. The air temperature decreases practically linearly until it becomes equal to that of the chamber walls (usually $18-20^{\circ} \mathrm{C}$ ). This period lasts $\approx 12 \mathrm{~min}$. during which the equivalent velocity of air updraft decreases to $\approx 0.9 \mathrm{~m} / \mathrm{s}$. Just during this period the process of cloud medium formation in the BCC may be considered adequate to the process of convective cloud formation in the real atmosphere.

As the total heat capacity of the BCC walls is by about 10 times greater than the total heat capacity of air in the chamber, the evolution of the cloud medium is strongly affected by the processes of air heat exchange with the chamber walls. When the air temperature becomes lower than that of the wall, the wall heats the air. Despite the continuing lowering of air pressure the rate of air temperature change in the BCC decreases. The total cooling of the air in the BCC reaches $\sim 7^{\circ} \mathrm{C}$ by this time. During the experiment the wall chamber temperature changes very slowly (no more than by $0.7^{\circ} \mathrm{C}$ ). After 20 minutes of cloud formation, the air temperature starts to increase and the cloud begins to evaporate. The total lifetime of the cloud in the BCC is $40-50 \mathrm{~min}$.

For measurements of cloud medium microstructure the $\mathrm{BBC}$ is equipped with the photoelectric particle analyzer developed at the RPA "Typhoon" (Romanov, 1991). This device is based on measurements of light scattered from particles at an angle of $90^{\circ}$. The principle of operation of the analyzers is in the analysis of amplitudes of pulses of light scattered from single particles at their flight through the mea- suring volume. An electric filament lamp is used as a white light source. The light flux from the lamp is focused by a round lens on its axis. The light scattered from a drop in the lens focus is collected by the round lens and directed to the photodetector. The angle between the axes of the illuminating and the positive lenses makes $90^{\circ}$. The principle of drop sizes measurements is in the measurement of the intensity of light scattered by a drop and the use of the calculated dependences of scattered light intensity on a drop size. For this, the operating characteristic of the device is calculated. The operating characteristics of such devices are calculated with the Mie scattering theory for spherical particles (Heyder and Gebhart, 1979; Singh et al., 1982). The angular apertures of the light source and of the light detector are taken into consideration. The lamp radiation spectrum and the photodetector spectral sensitivity are also taken into account. The range of measured drop radii with this meter is within $1-50 \mu \mathrm{m}$. The number of drop size measuring channels is 360 . The photoelectric meter allows one to obtain cloud drop spectra at the time of data accumulation from $1 \mathrm{~s}$ at the medium flow rate through the measuring volume over $2.5 \mathrm{~cm}^{3} / \mathrm{s}$, which was driven through the probe by a fan located downstream of the measurement volume. During the measurements in the BCC the photoelectric meter was located near the bottom of the cloud chamber.

For measurements of dry aerosol particles microstructure a laser analyzer of particles developed at the RPA "Typhoon" (Kolomiets at al., 1989) is used. A continuously operating laser at the $0.63 \mu \mathrm{m}$ wavelength serves as a light source in this analyzer. High uniformity of illumination of the measuring volume is achieved in the laser analyzer because of the use of an annular laser beam and subsequent electronic processing of photoelectric pulses. The annular beam is formed from the initial laser beam with the help of a special lens (axicon) having one flat surface and the other one is conical in form. The light scattered by particles under study is collected with the elliptical mirror and sent onto a photodetector in the frames of the annular aperture in the scattering angles of $60^{\circ}-120^{\circ}$. The operating characteristic of the laser analyzer is calculated with the account for the geometric peculiarities of its optical scheme. The laser analyzer makes it possible to measure particle size distributions within the particle radii of $0.1-5 \mu \mathrm{m}$. The number of particle size measurement channels is 120 . The aerosol under study is supplied via a thin capillary into the region of laser beam focusing. The flow rate of air passing through the analyzer volume is $1 \mathrm{~cm}^{3} \mathrm{~s}^{-1}$.

In both devices described above electric signals from the photodetectors, proportional to the intensity of light scattered from a separate light particle, go to the amplitude pulse analyzer and then to the computer. In the computer, the particles sizes are calculated according to the operating characteristic of the analyzers and the particles size distribution function is constructed. The particle concentrations are determined from the frequency of pulses of light scattered from every particle. 
The integral parameters of the size distribution function such as mass concentrations and effective radii of the particles are also calculated from the analyzer data. Both photoelectric analyzers are calibrated against round polysterol latex particles with the known sizes and the known light refraction index.

The experiments in the BCC on cloud medium modification by the salt powder were performed according to the following method. First a cloud medium on natural condensation nuclei was formed in the BCC, and during about 20 min measurements of the background cloud medium parameters were made. Then the pumping of the chamber up to the initial high pressure was repeated and salt particles were injected into it. The spraying of the salt powder from an airplane in the cloud was simulated by a pneumatic atomizing nozzle (like a spraying can). With the help of fans the aerosol was uniformly mixed inside the chamber. At the moment of the beginning of pressure decrease the fans are switched off. The salt particles were introduced into the chamber at a relative humidity of about $96 \%$. By this, the conditions of particles introduction into the subcloud layer of a convective cloud were simulated. Then, the process of lowering air pressure in the chamber was repeated, and the parameters of the cloud medium formed as a result of aerosol particles impact were measured. The modification effect was assessed from the results of comparing the parameters of the cloud medium with the corresponding parameters during the background experiment. During numerous experiments in the BCC it has been found that at repeated lowering of air pressure the parameters of the cloud medium remain practically the same, so the impact of salt particles with the use of the method developed is made under similar conditions of cloud medium formation as compared to the background experiment.

The agent under study is a specially prepared powder of $\mathrm{NaCl}$ with aerosil $\left(\mathrm{SiO}_{2}\right)$ used as an anticaking admixture (Lahav and Rosenfeld, 2005; Rosenfeld et al., 2010). The powder was designed to reproduce the optimal size distribution for most efficient acceleration of warm rain processes, as calculated by Segal et al. (2004). The measurements of microstructure of the dry salt particles under this study were made in the BCC at the air relative humidity of about $40 \%$ with the laser analyzer of particles. After the salt powder was introduced into the chamber with the pneumatic atomizing nozzle the aerosol was uniformly mixed inside the chamber and measured there. The results of measurements of the dry particle size distribution function of the salt powder are shown in Fig. 2. Here also given are the spectra of background aerosol the mass concentration of which made $5 \times 10^{-4} \mathrm{mg} / \mathrm{m}^{3}$. The measurements were performed at a salt mass concentration of $0.4 \mathrm{mg} / \mathrm{m}^{3}$. The spectra are obtained during the time of data accumulation of about $10 \mathrm{~min}$. The spectrum of salt powder particles was obtained by subtraction of background aerosol spectrum from the aerosol spectrum in the BCC after the introduction of the salt powder. As is seen from the graphs, in the salt powder studied there

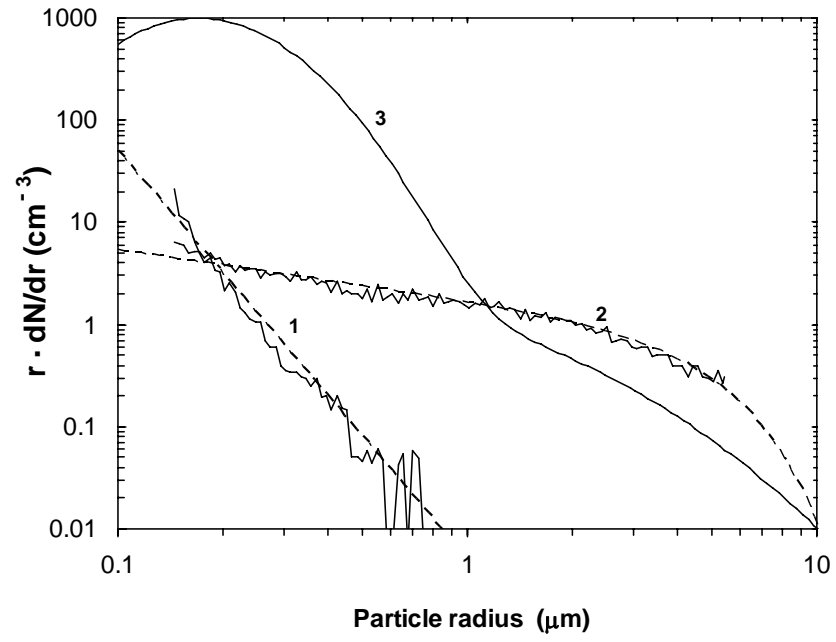

Fig. 2. Observed background aerosol particles spectrum in the BCC at a mass concentration of $5 \times 10^{-4} \mathrm{mg} / \mathrm{m}^{3}(1)$, the spectrum of dry salt particles (2) and the spectrum of the South African hygroscopic flares particles (3) both at the same mass concentration $0.4 \mathrm{mg} / \mathrm{m}^{3}$. Dashed lines refer to the approximation made by functions $(1,2)$.

is a considerable number of particles the sizes of which are smaller than the lower measurement limit of the laser analyzer $(0.15 \mu \mathrm{m})$. These may be the particles of the anticaking admixture or the "fragments" of salt. The particles effective radius in the size range studied is $1.54 \mu \mathrm{m}$. As is seen from Fig. 2, there are also particles with the sizes exceeding the upper measurement limit of the laser analyzer $(5 \mu \mathrm{m})$. To reveal the structure of these particles, aerosol particles sampling was made by their settling onto a glass substrate during the measurements in the BCC. Microscopic analysis of the studied powder have shown the occurrence of large particles with the radii up to $10 \mu \mathrm{m}$. The particles with sizes over $5 \mu \mathrm{m}$ are mainly salt conglomerates with finer particles that stuck to them. It should be noted here that the salt powder was tested three years after it was manufactured and held in closed plastic bags within cardboard boxes in a storage room, so that some clumping could take place during this long period.

Figure 2 gives the approximation of background aerosol particles spectrum made by the Junge spectrum

$f(r)=0.005 r^{-v}$,

where $v=5$. The spectrum of salt particles, as is seen from Fig. 2, is rather well approximated by the distribution like

$f(r)=1.7 r^{-1.5} \exp \left(-\left(r / r_{o}\right)^{2}\right)$,

where $r_{o}=5 \mu \mathrm{m}$.

For comparison, the particle size distribution of the South African hygroscopic flares (Cooper et al., 1997) is given in Fig. 2 at a mass concentration equal to the salt powder concentration of $0.4 \mathrm{mg} / \mathrm{m}^{3}$. Figure 2 reveals that the South 


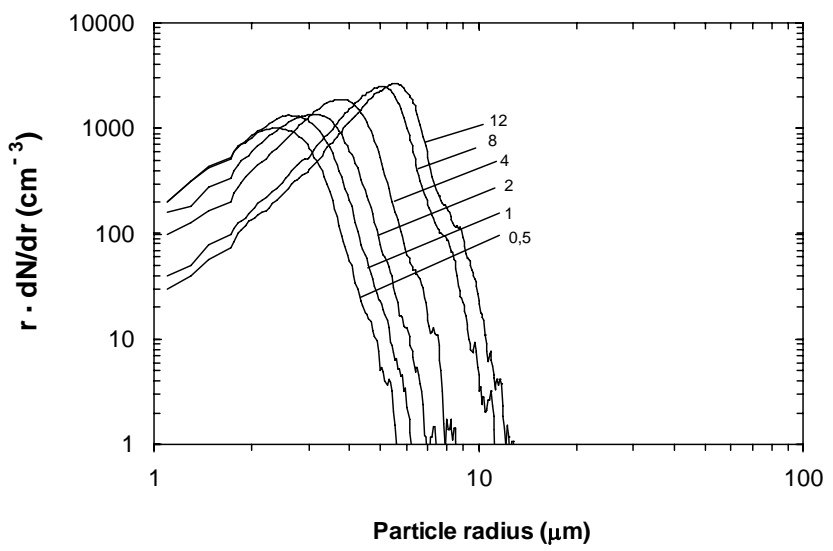

Fig. 3. Observed evolution of cloud drop spectrum in the background experiment (numbers at the curves - time (min.) after the cloud medium formation).

African flares contain a very high number of particles with $r<1 \mu \mathrm{m}$ as compared to the salt powder. At the same time the particles with $\mathrm{r}>1 \mu \mathrm{m}$ are much less in number.

\section{BCC measurements of salt seeding effects on cloud microstructure}

As it has been mentioned above, in the BCC the initial stage of convective cloud microstructure formation is modeled. The simulation of the raindrop embryo effect is likely to be impossible. But, as it is shown in (Drofa, 2006), the transformation of a cloud drop spectrum as a result of the introduction of hygroscopic particles at the initial stage of cloud formation plays the crucial role in the effect of modification. The size spectrum of the droplets formed in the initial stage of cloud origination determines the subsequent development of cloud microstructure, rate of large drop formation, development of coagulation processes and precipitation formation. This Section presents the measurement results of changes of the cloud microstructure in the cloud chamber at the introduction of the salt powder and the estimations of the impact of the salt powder based on the observed changes of the cloud microstructure.

The evolution of the cloud drop size spectra obtained with the use of a photoelectric meter in the BCC is shown in Fig. 3. Here presented are typical cloud drop spectra determined at different time steps after the formation of a cloud medium during the background experiments. The time starts from the moment of the cloud medium formation. At the chosen mode of air pressure lowering a cloud medium with the drop concentration from 1300 to $1550 \mathrm{~cm}^{-3}$ is formed. The liquid water content of the medium grows linearly with time and by $12 \mathrm{~min}$ it becomes equal to $0.8 \mathrm{~g} / \mathrm{m}^{3}$. The largest drops in the background experiments reached the radii of $12 \mu \mathrm{m}$.

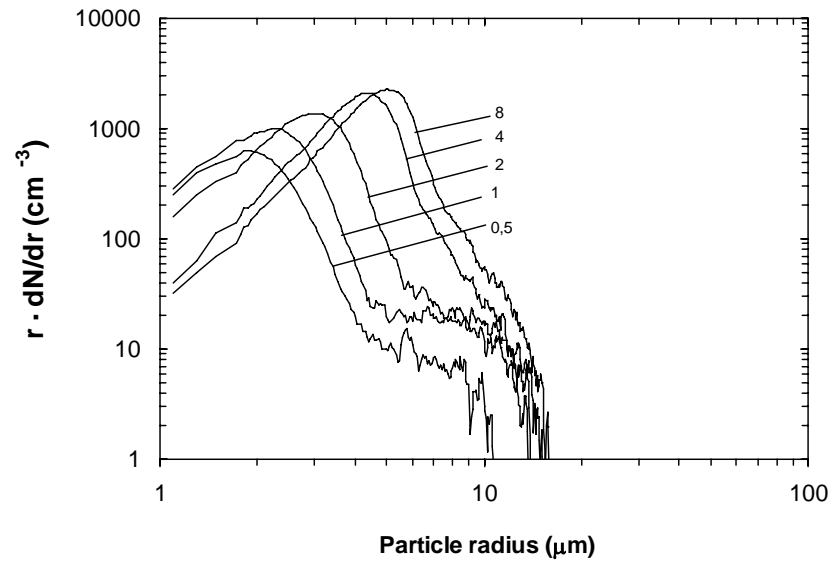

Fig. 4. Observed evolution of cloud drop spectrum at introduction of salt powder into the BCC with the mass concentration of $1.1 \mathrm{mg} / \mathrm{m}^{3}$ (numbers at the curves - time (min.) after the beginning of cloud medium formation).

Figure 4 gives the results of cloud drop spectrum measurements at different time steps at the introduction of $3.5 \mathrm{~g}$ of the salt powder into the BCC. When distributed over the BCC volume of $3200 \mathrm{~m}^{3}$ it constitutes a mass concentration of $1.1 \mathrm{mg} / \mathrm{m}^{3}$. The modal size of the cloud drops that formed in the BCC appear to be nucleated on the background condensation nuclei. The temporal character of variation of this spectrum range does not practically differ from the behavior of spectra obtained during the background experiment. From the graphs in Fig. 4 it is seen that the introduction of such an amount of salt particles manifests itself only in the large-drop fraction of the cloud drop spectrum. A "tail" of large drops that formed on the added salt particles appeared on the drop size distribution that was measured already at the first 2 minutes after cloud formation. In the spectra measured after 4 minutes of the cloud medium existence the large-drop "tail" of the distribution appears to be truncated due to gravitation-induced sedimentation of the largest drops onto the BCC floor. Actually, the terminal sedimentation rate of drops with the radii of, for example, $30 \mu \mathrm{m}$, is equal to $11 \mathrm{~cm} / \mathrm{s}$. At the height of the BCC working volume of 15 meters all the drops of such sizes formed at this level fall onto the floor of the BCC during $2.5 \mathrm{~min}$. The duration of the settling process of drops with radii of $20 \mu \mathrm{m}$ takes about 5 minutes. It is clear that the life-time of such drops formed at the lower levels of the BCC is much shorter. This causes a sharp decrease of the observed number of drops with radii larger than $20 \mu \mathrm{m}$. The effective radius of drops calculated over the spectra increased in this case by $5 \%$ as compared to the background experiment, and the largest registered drops reached radii of $16 \mu \mathrm{m}$.

The evolution of cloud medium spectra at the introduction of $16 \mathrm{~g}$ of salt particles with the mass concentration of $5 \mathrm{mg} / \mathrm{m}^{3}$ is shown in Fig. 5. Here the bimodal character of 
Table 1. Measurement results of cloud medium parameters in the experiments of introduction of salt powder particles into the cloud chamber.

\begin{tabular}{lllllllll}
\hline $\mathrm{N}$ & Salt mass $(\mathrm{g})$ & Mass concentration $\left(\mathrm{mg} / \mathrm{m}^{3}\right)$ & $N_{B}\left(\mathrm{~cm}^{-3}\right)$ & $N\left(\mathrm{~cm}^{-3}\right)$ & $N_{B} / N$ & $r / r_{B}$ & $S_{B}$ & $S$ \\
\hline 1 & 1.0 & 0.31 & 1380 & 1370 & 1.00 & 1.00 & 0.285 & 0.287 \\
2 & 3.5 & 1.1 & 1400 & 1220 & 1.15 & 1.05 & 0.283 & 0.289 \\
3 & 11 & 3.4 & 1290 & 820 & 1.57 & 1.16 & 0.286 & 0.320 \\
4 & 16 & 5.0 & 1300 & 710 & 1.83 & 1.22 & 0.276 & 0.326 \\
5 & 21 & 6.6 & 1340 & 680 & 1.97 & 1.25 & 0.290 & 0.334 \\
6 & 31 & 9.7 & 1560 & 630 & 2.48 & 1.35 & 0.284 & 0.367 \\
7 & 35 & 10.9 & 1430 & 490 & 2.92 & 1.43 & 0.290 & 0.391 \\
\hline
\end{tabular}

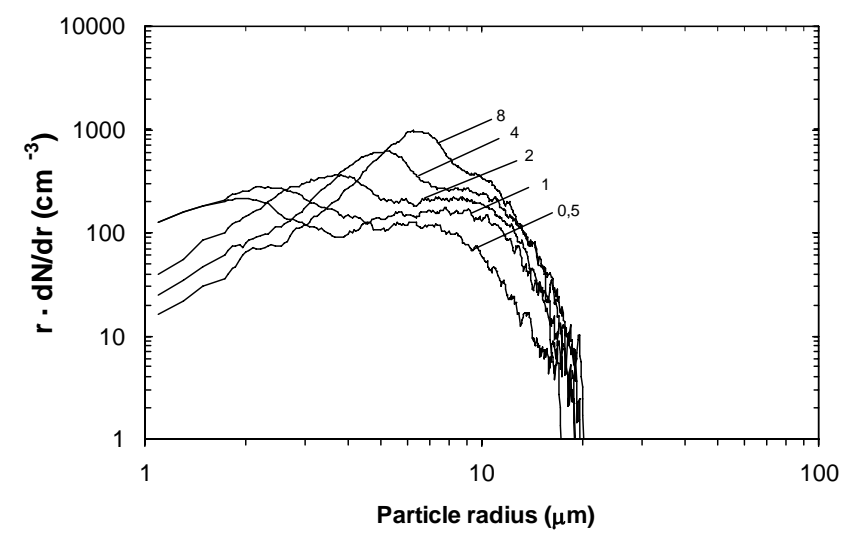

Fig. 5. Observed evolution of cloud drop spectra at the introduction of salt powder into the BCC with the mass concentration of $5 \mathrm{mg} / \mathrm{m}^{3}$ (numbers at the curves - time (min.) after the beginning of cloud medium formation).

the cloud drop spectrum is seen distinctly. In this case the cloud droplets formed on the background aerosol particles are smaller in size as compared to the background experiment. This effect is explained by a decrease of water vapor supersaturation in the cloud medium at the introduction of the given amount of salt particles. As a result, a slower growth of drops formed on the background aerosol occurs. The fraction of large cloud drops formed on the salt particles is here significantly greater than in the previous case. The drop effective radius at the introduction of $16 \mathrm{~g}$ of salt powder increased as compared to the background experiment by $20 \%$. The largest drops attained radii of $20 \mu \mathrm{m}$.

At the introduction into the $\mathrm{BCC}$ of a rather great amount of salt particles $(31 \mathrm{~g}$, with the mass concentration of $9.7 \mathrm{mg} / \mathrm{m}^{3}$ ), as is seen from Fig. 6, practically all the cloud drops are formed on salt particles. The fine-droplet spectrum fraction, that appeared due to the background condensation nuclei, does not manifest itself. The effective radius of drops increased in this case as compared to the background experiment by 1.35 times, and the largest drops registered reached the radii of $22 \mu \mathrm{m}$. The cloud drop concentration here decreased by 2.5 times. An example of changes in the integral

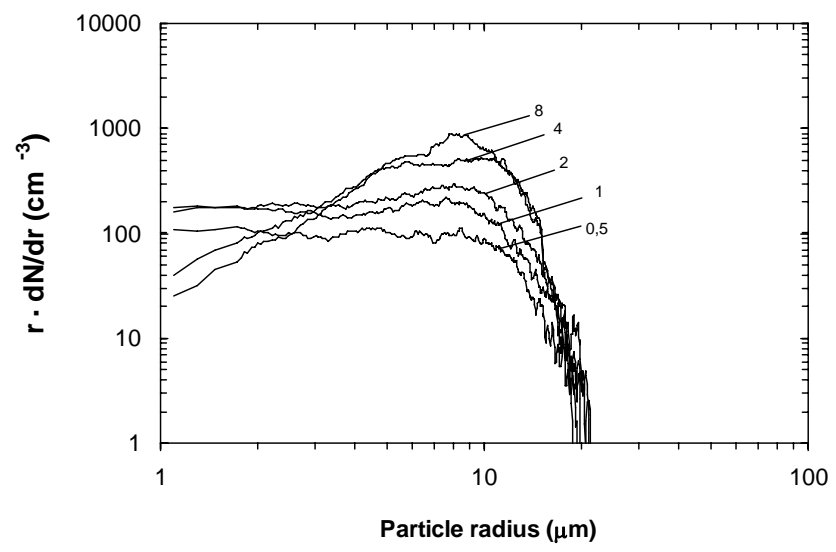

Fig. 6. Observed evolution of cloud drop spectra at the introduction of salt powder into the BCC with the mass concentration of $9.7 \mathrm{mg} / \mathrm{m}^{3}$ (numbers at the curves - time ( $\mathrm{min}$ ) after the beginning of cloud medium formation.

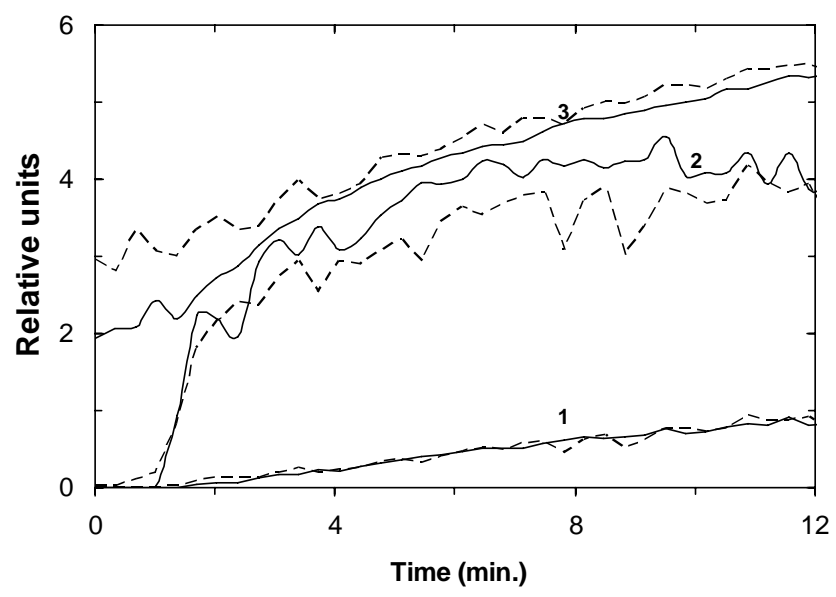

Fig. 7. Observed changes with time of cloud medium liquid water content $\left(1, \mathrm{~g} / \mathrm{m}^{3}\right)$, concentration $\left(2,300 \mathrm{~cm}^{-3}\right)$ and effective radius of cloud drops $(3, \mu \mathrm{m})$ in the background experiment (solid curves) and at the introduction of salt powder with the mass concentration of $1.1 \mathrm{mg} / \mathrm{m}^{3}$ (dashed curves). 
parameters of cloud medium microstructure during the background experiment and in the experiment in the $\mathrm{BCC}$ with the introduction of $3.5 \mathrm{~g}$ of salt particles is presented in Fig. 7 . The time count begins at the moment of the cloud medium formation. As one can see from the data presented, the introduction of the salt powder results in the formation of larger cloud drops and their lower concentrations as compared to the background experiment. The cloud medium liquid water contents are practically the same in both experiments. The experimental results have shown that at an increase of the mass of the particles introduced, the modification effect becomes stronger.

The results of measuring the cloud medium integral parameters at different masses of particles introduced are given in Table 1. Table 1 contains the salt particle mass concentrations at different masses of salt powder introduced, and cloud drop concentrations at the introduction of particles $(N)$ and in the background experiment $\left(N_{B}\right)$. Table 1 gives averaged data obtained during the experiment in $4-5 \mathrm{~min}$. after the cloud medium formation in the chamber, i.e. after the process of nuclei activation ended and the cloud medium parameters are stabilized. It contains also the values of a relative dispersion of cloud drop spectra at the introduction of particles $(S)$ and in the background experiment $\left(S_{F}\right)$ calculated from the measured spectra with the formula $S=\sqrt{\left(r_{2} / r_{1}\right)^{2}-1}$, where $r_{1}$ and $r_{2}$ are the mean and mean-root-square radii of drops, respectively. The values of $S$ are calculated for the 12th minute of the cloud medium existence.

A necessary condition for obtaining a positive effect at modification by hygroscopic particles (precipitation enhancement) is an increase of cloud drop sizes at the introduction of particles (Drofa, 2006), as far as the enlargement of cloud drops is the major factor stimulating gravitational coagulation in clouds and subsequent precipitation formation. Changes of cloud drop sizes were estimated by us during modification experiments over the changes of their concentrations against the drop concentrations in the background experiment. Under similar conditions of performing these experiments (the same liquid water contents of cloud media) the relationship $N_{B} / N=\left(r / r_{B}\right)^{3}$ is valid, where $r$ and $r_{B}, N$ and $N_{B}$ are the effective radii of drops, their concentrations at modification and in the background experiment correspondingly. It means that the value of $N_{B} / N$ characterizes the changes of cloud drop sizes under modification. It is easily measured experimentally. In such a way the values of $r / r_{B}$ given in the Table 1 were obtained.

The effect of modification can be considered positive at $N_{B} / N>1$ (i.e. when the drop become larger). As one can see from the data given in the Table, the necessary condition for obtaining a positive effect of modification with the salt powder under study in our experiments is met - at the introduction of salt particles a decrease of cloud drop concentration and the drop growth are observed. With increasing mass concentration of salt particles introduced, this effect of mod- ification increases practically linearly. The introduction of a great amount of salt particles does not result in "overseeding" at which the concentration of drops increases and instead of their enlargement the drops become smaller.

The measurement results in a relative droplet size dispersion (given in Table 1) that shows that the introduction of salt particles in the cloud medium causes the spectrum to be broader than in the background cloud. This effect increases with increasing the particle mass. The effect is a positive factor at cloud medium modification for precipitation enhancement as far as the greater the difference in drop sizes in the cloud medium, the more efficient the coagulation processes of cloud drops and precipitation formation are. This is the "tail effect", as defined in the introduction. As has been mentioned earlier, the drop spectra obtained in the BCC are "truncated" in the large-drop-spectrum range because of the settling of drops onto the BCC floor, so that the "rain embryo" effect cannot be properly simulated in the experiment. It is most probable that in real clouds broadening of cloud drop spectra may be considerably larger than in the experiment.

The value of $N_{B} / N$ can serve as an estimate of the efficiency of the competition effect for cloud modification with hygroscopic particles having relatively narrow particle size distributions (Drofa, 2006). At the introduction of such particles into the subcloud layer governing in the stimulation of coagulation processes in a cloud is the increase of the whole population of cloud drops. The more the value of $N_{B} / N$ is realized in the cloud base, the greater will be the amount of additional precipitation at modification. A characteristic property of modification by particles with narrow drop size distributions is that the positive effect of modification is realized only at an optimal concentration of such particles. More than $1 \mathrm{mg} \mathrm{m}^{-3}$ of salt powder is required for starting the competition effect with $N_{B} / N>1.1$. This means that when dispersed homogeneously at this concentration, $1 \mathrm{~kg}$ of salt powder would fill a cloud volume of $10^{6} \mathrm{~m}^{3}$ or $10^{-3} \mathrm{~km}^{3}$. Actual measurements of dispersion of seeding material showed a dispersion greater by a factor of $10^{3}$ at $1 \mathrm{~km}$ above cloud base (Rosenfeld et al., 2010) leading to a respectively smaller concentrations of the seeding agent. Furthermore, as will be shown in the next section, the tail and rain embryo effects of the salt powder totally dominate the rain production at the initial concentrations where $N_{B} / N>1$.

An example of the use of hygroscopic flare particles is the modification of convective clouds by pyrotechnic flares developed in South Africa (Mather et al., 1997). Their particle size distribution is given in Fig. 2. When such flares were used, the positive meaningful modification effect was obtained in several projects aimed at obtaining additional precipitation amounts from convective clouds. Using the method proposed in (Drofa, 2006) with the known data on particle size distribution, physical and chemical characteristics of pyrotechnic flares for typical atmospheric conditions, it is possible to obtain a maximum value of $N_{B} / N=1.42$. This value is achieved at a certain 
concentration of particles introduced $\left(300-500 \mathrm{~cm}^{-3}\right)$. The mass concentration of the substance contained in pyrotechnic flares makes $\approx 0.05 \mathrm{mg} / \mathrm{m}^{3}$. At higher or lower concentrations the value of $N_{B} / N$ decreases. When mass concentrations of particles introduced make over $0.14 \mathrm{mg} / \mathrm{m}^{3}$, an effect of "overseeding" appears, i.e. at the introduction of particles the drop concentration in the cloud increases and their mean size decreases. This is the cause of decreasing intensity of precipitation formation in the cloud at "overseeding".

\section{Simulations of the seeding effects}

To overcome the effect of truncation of the drop size distribution by sedimentation and to assess the efficiency of hygroscopic agents with rather wide particle size distribution functions (one of them is the salt powder under study), simulation studies are needed. In this paper we shall theoretically study the efficiency of cloud modification by the salt powder with the use of a one-dimensional convective cloud model developed by Drofa $(2007,2008,2010)$. In these articles, an analysis of evolution of cloud microstructure and precipitation in response to the introduction of hygroscopic particles is made, and the efficiency of hygroscopic seeding of clouds is studied depending on cloud parameters and physicochemical properties of the hygroscopic agent. Similar studies made with the use of a 3-D model are made in the recently published paper (Kuba and Mukarami, 2010). The results of this work are in complete agreement with the conclusions drawn earlier. As in all the works cited in the given paper that concern numerical simulation of hygroscopic seeding of clouds, the 1-dimensional numerical model presented does not consider ice processes. Therefore, the conclusions are limited to the effects of hygroscopic aerosols on initiating warm rain. In tropical and sub-tropical summer convective clouds the distance between cloud base an the $0{ }^{\circ} \mathrm{C}$ isotherm level can reach $4 \mathrm{~km}$, with additional $1-2 \mathrm{~km}$ of supercooled cloud above this level, where precipitation initiation is dominated by warm rain processes.

The 1-dimensional numerical model describes the evolution of a cloud medium in the central part of an axisymmetric warm convective cloud at a preset variable with height velocity of an air updraft forming the cloud. The equation system is used for temperature and air pressure changes and for water vapor supersaturation at air mass lifting. Entrainment of heat and water vapor into a lifting air parcel from the environment is accounted for parametrically (as in Pruppacher and Klett, 1997). The value of the entrainment coefficient is taken inversely proportional to the altitude above the cloud base. Adjustment of parameters characterizing entrainment gives a possibility to achieve a complete matching of vertical profiles of cloud parameters obtained in the model with the parameters of continental clouds in the real atmospheric conditions (Mazin and Shmeter, 1983; Shmeter, 1987).
The vertical profile of vertical air velocity is prescribed in the numerical model and does not change between the stages of cloud development. To describe the air updraft velocity vertical profile a universal function considering basic regularities of air vertical fluxes in continental convective clouds (Shmeter, 1987) is applied. The velocity of an air updraft at the cloud base is set to $1.5 \mathrm{~m} / \mathrm{s}$. It increases proportionally to altitude in the lower half of the cloud. Maximum velocity is achieved at the half cloud height. It is proportional to the cloud thickness. At a cloud thickness of $4 \mathrm{~km}$ it reaches about $5 \mathrm{~m} / \mathrm{s}$. In the cloud upper half the updraft velocity drops gradually to zero. The altitude at which the updraft velocity is equal to zero is determined by the cloud thickness.

Limitation of growth of the convective cloud upwards occurs due to the existence in the atmosphere of a barrier layer with isothermality and inversion of air temperatures. The height of this layer above the cloud base determines the height of maximum liquid water content in the cloud. In the numerical model, the atmospheric barrier layer is simulated by the introduction at a certain level above the cloud base of water vapor sub-saturation, as far as low air relative humidity has a governing role in the cloud medium evolution. The altitude of the barrier layer above the cloud base is preset at the level of 0.8 of the cloud thickness. This corresponds to the parameters of moderate convective clouds observed in real atmospheric conditions. Above this level the cloud top is formed, where fine cloud droplets evaporate and the large drops precipitate into the lower cloud layers.

In the given numerical model it is assumed that the activation of condensation nuclei takes place in the cloud base. At a further lifting of the cloud medium only the process of drop condensation growth/evaporation occurs. New cloud drops do not form. The initial stage of cloud medium microstructure formation is described by the equation of drop condensation growth. With the use of the initial conditions of the air mass state and the parameters of condensation nuclei (atmospheric and/or additionally introduced), the size distribution function of drops originated at the cloud base is calculated according to the method used in (Drofa, 2006). Further evolution of the size distribution function is calculated with the use of the kinetic equation. The model contains a precise description of warm rain microphysics. The processes of drop condensation growth/evaporation, coagulation, breakup of drops and their sedimentation are considered. For a numeric solution of the kinetic equation a fixed grid of cloud drop sizes in the radius range of $1-2500 \mu \mathrm{m}$ (396 points) with a non-uniform step in radii was used. Sedimentation of drops and precipitation are computed from the difference of terminal falling velocities of drops of different radii and the rate of the air mass updraft. For this determined is the number of drops that fell from the given cloud layer and those that entered this layer from the above level during a certain period of time.

With the data obtained during model simulation calculated are the spatiotemporal structure of meteorological 


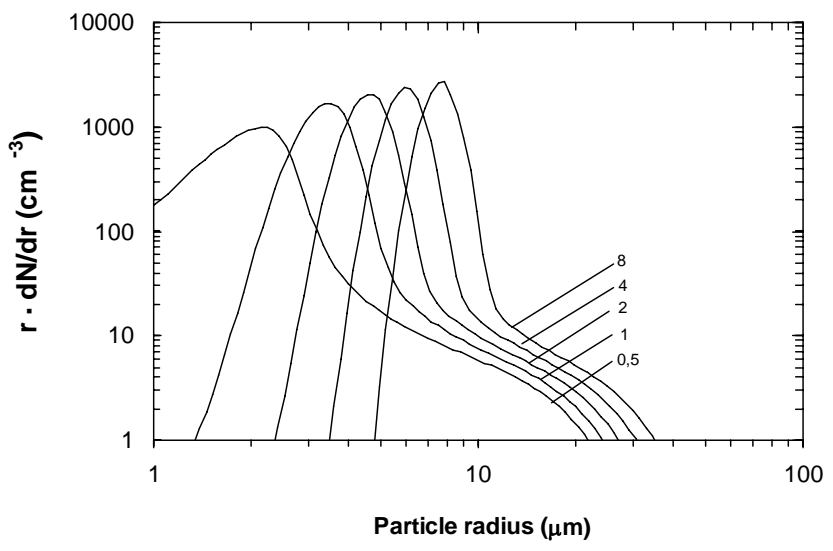

Fig. 8. Evolution of simulated cloud drop spectrum at the introduction of salt powder with the mass concentration of $1 \mathrm{mg} / \mathrm{m}^{3}$ (the numbers at the curves - time (min.) after the beginning of the cloud medium formation).

parameters and integral characteristics of the cloud drop size distribution function - the drop number concentration, cloud liquid water content and the cloud drop effective radius. The liquid water contents of large drops with the radii $r>200 \mu \mathrm{m}$ characterizing precipitation are computed as well. Total amounts of precipitation are calculated at the level of the lower cloud boundary. It should be said that owing to the fairly simplified description of the dynamic structure of the cloud development, dynamic responses of the clouds to the modified precipitation are not considered in the model. The results of calculated precipitation in this model should be regarded as relative estimates. They can be used only for comparison of the efficiencies of the tested hygroscopic cloud seeding methods.

An example of computation of the initial stage of the cloud medium microstructure formation at the introduction of the salt powder into the subcloud layer of the convective cloud according to the method used in (Drofa, 2006) is shown in Fig. 8. Here presented are the calculation results of cloud drop spectrum evolution with time for the conditions typical of continental convective clouds. The physical and chemical properties of the aerosol correspond to mean characteristics of atmospheric aerosol of continental origin (Drofa, 2006). The Junge distribution (1) was used as an initial distribution of aerosol particles being atmospheric condensation nuclei at $v=4$ (see Fig. 2). The velocity of air mass updraft is accepted as $V=1.5 \mathrm{~m} / \mathrm{s}$. The initial temperature of air is $10^{\circ} \mathrm{C}$. The pressure is $900 \mathrm{hPa}$. To describe the salt particle size distribution, shown in Fig. 2, the function like (2) was used. It corresponds to experimentally measured spectra of dry salt powder particles. The sizes of salt particles were accounted for within the range of radii from 0.01 to $10 \mu \mathrm{m}$. The mass concentration of the particles introduced was taken equal to $1 \mathrm{mg} / \mathrm{m}^{3}$. At such a concentration, as is seen from Fig. 8, the effect of salt particles reveals itself only in the large drop "tail" of cloud drop distribution range without changes in the spectrum of drops formed on atmospheric condensation nuclei. The same character of cloud drop spectrum variations is observed in experimentally obtained spectra measured in the $\mathrm{BCC}$ at a very similar concentration of the salt powder introduced (see Fig. 4). The difference is observed only toward smaller droplet sizes - the experimental spectra are broader. On the whole, the problem "to broaden spectra" needs special detailed studies. One of possible causes of such a discrepancy may be in differences of physical and chemical characteristics of atmospheric condensation nuclei in particle size distributions. In the $\mathrm{BCC}$ only the size distributions of all aerosol particles were measured. But this distribution can concern a mixture of particles with rather different properties.

The calculation results of drop spectra in the convective cloud after $120 \mathrm{~s}$ at the introduction of different amounts of the salt powder into the subcloud layer are presented in Fig. 9. The atmospheric conditions are the same as in the case mentioned above. As it is seen from Fig. 9, at a small mass concentration of the powder, the introduction of such particles results only in the growth of the large-cloud drop fraction. This drop fraction is growing with increasing mass of the powder introduced. The shape of the drop spectrum formed on the background aerosol particles does not change at their mass concentration of the particles up to $1 \mathrm{mg} / \mathrm{m}^{3}$.

At the concentrations of the salt powder higher than $1 \mathrm{mg} / \mathrm{m}^{3}$ a decrease of the number of drops formed on atmospheric condensation nuclei occurs. This effect is also observed in the experimental data obtained at high concentrations of the salt powder (see Figs. 5 and 6 and Table 1). The effect is explained, as it has been mentioned earlier, by the fact that the introduction of larger amounts of salt particles decreases the supersaturation of water vapor. This causes a slower growth of drops formed on the background aerosol particles.

The evolution of the cloud drop size spectrum at the introduction of hygroscopic particles with a very narrow particle size distribution into the convective cloud sublayer is given as an example in Fig. 9 as well. In this case, the particles of $\mathrm{NaCl}$ with an effective radius of $1 \mu \mathrm{m}$ and a relative dispersion of the drop size spectrum $S=0.3$ are introduced. The number concentration of the particles introduced is $120 \mathrm{~cm}^{-3}$. This is an optimal concentration of particles of the given sizes for obtaining maximum effect of modification (Drofa, 2008). At higher or lower concentrations the modification effect decreases. The particle mass concentration is $1.1 \mathrm{mg} / \mathrm{m}^{3}$. As is seen from Fig. 9, the result of the introduction of such particles is in the formation of a bimodal cloud drop spectrum. The large drop mode is governed by the growth of drops formed on salt particles. The small drop mode is formed on the background aerosol particles. Further, the bimodal character of the cloud drop spectrum is maintained in the quasi-equilibrium state. 


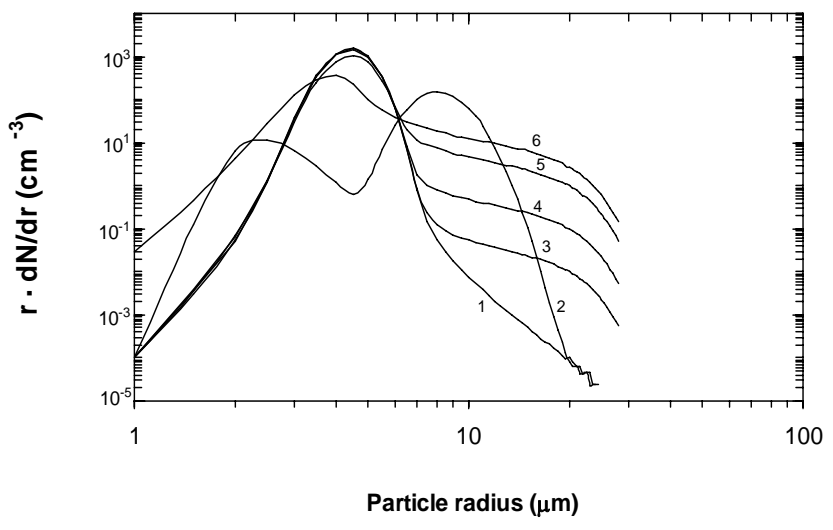

Fig. 9. Simulated drop spectrum in the background cloud (1), at the introduction of salt particles with the radius of $1 \mu \mathrm{m}$ with the mass concentrations of $1.1 \mathrm{mg} / \mathrm{m}^{3}(2)$ and at the introduction of salt powder with the mass concentrations of $0.01,0.1,1$ and $5 \mathrm{mg} / \mathrm{m}^{3}$ (curves 3-6, correspondingly).

The studies of the modification effect induced by such particles have shown (Segal et al., 2004, 2007; Drofa, 2006) that the introduction of salt particles can accelerate warm rain processes by the competition effect, which decreases the water vapor supersaturation in the cloud. In turn, the number of atmospheric nuclei is activated (i.e. they turn into cloud drops) to a lesser degree than in the background not seeded cloud. As a result, the total concentration of cloud drops formed on the background and additional seeded nuclei appears less than in the case when additional seeded particles are absent. Average sizes of the seeded cloud drops become larger. Due to this, a positive effect of modification by hygroscopic particles on a cloud with narrow drop size distributions is attained, because the enlargement of cloud drops is the major factor stimulating gravitation-induced coagulation in clouds and subsequent precipitation formation. One should pay attention to the fact that the cloud drops formed on the background aerosol particles in the seeded cloud are smaller in size as compared to those in the not seeded cloud. It means that in this case the impact of salt particles leads to changes in the conditions of cloud drop formation on background condensation nuclei.

Modification made by the salt powder does not result in such changes even at rather high mass concentrations of the powder introduced. The number of cloud drops formed on background aerosol particles changes little here. As the analysis of the results of numerical simulations demonstrate, the modification by the salt powder causes a higher intensification of coagulation processes in the cloud than at the modification by hygroscopic particles with narrow size distributions. This can be explained by the fact that at the modification by the salt powder the drop spectrum is broadened only towards the large-drop fraction where coagulation is more efficient.
The data obtained at the initial stage of condensation (Fig. 9) are used as starting data for calculations of evolution of cloud medium microstructure with the one-dimensional numerical model of a convective cloud (Drofa, 2010). The calculation results for precipitation obtained with the model for clouds of different thicknesses at modification by different amounts of salt particles are shown in Fig. 10. The introduction of particles into the 60,120 or $240 \mathrm{~m}$ subcloud layer is made at the 10th min. from the beginning of the cloud formation. The data given in the same figure for clouds without modification demonstrate that the significant precipitation quantities calculated with this model fall out from convective clouds with thicknesses over $3.5 \mathrm{~km}$. This result is in agreement with the data of experimental studies of clouds in real atmospheric conditions (Mazin and Shmeter, 1983).

The results of numerical simulations show that the effect of modifying clouds with hygroscopic particles significantly depends on the cloud vertical thickness - the more the thickness, the greater the precipitation amounts are. The calculation data on the effect of the South African pyrotechnic flares particles show that significant additional precipitation amounts are observed only at modifying clouds with the thicknesses over $4 \mathrm{~km}$. The calculations are made for the case of particles introduction into the layer with the thickness of $240 \mathrm{~m}$ at a mass concentration of particles of $0.05 \mathrm{mg} / \mathrm{m}^{3}$ and the consumption of $12 \mathrm{~kg}$ of the agent per $1 \mathrm{~km}^{2}$ of the seeded area. The use of smaller amounts of such particles does not lead to a discernible positive modification effect. The result presented is in agreement with the data of field experiments aimed at cloud modification with such particles (Mather et al., 1997; WMO, 2000; Bruintjes et al., 2001). In these experiments at comparable with the above-mentioned agent consumptions a $10-15 \%$ precipitation enhancement from the clouds of 6-km thicknesses was obtained.

Modeling was made for different mass concentrations of the salt powder with $0.01,0.05,0.1$ and $0.2 \mathrm{mg} / \mathrm{m}^{3}$. The results of numerical simulations demonstrated that the effect of modification by the salt powder is determined by the total amount of the powder introduced into subcloud layer. This means that at the introduction of the powder with the concentration of, for example, $0.1 \mathrm{mg} / \mathrm{m}^{3}$ into the 120 -m layer the same precipitation amount is observed as at the introduction of the powder with the concentration of $0.2 \mathrm{mg} / \mathrm{m}^{3}$ into the $60-\mathrm{m}$ layer. The amount of the agent at the given mass concentration is determined by the thickness of the layer into which the agent is introduced. So, at the introduction of the powder with the particle mass concentration of $0.1 \mathrm{mg} / \mathrm{m}^{3}$ into the $120-\mathrm{m}$ layer the consumption of the agent is $12 \mathrm{~kg}$ per $1 \mathrm{~km}^{2}$ of the seeding area. At changing the layer thickness where the agent is introduced the agent consumption changes proportionally with the thickness of the layer.

As is seen from Fig. 10, seeding with salt powders results in a significant increase of precipitation amounts as compared to cloud modification by fine particles of the South African pyrotechnic flares. At the consumption of 


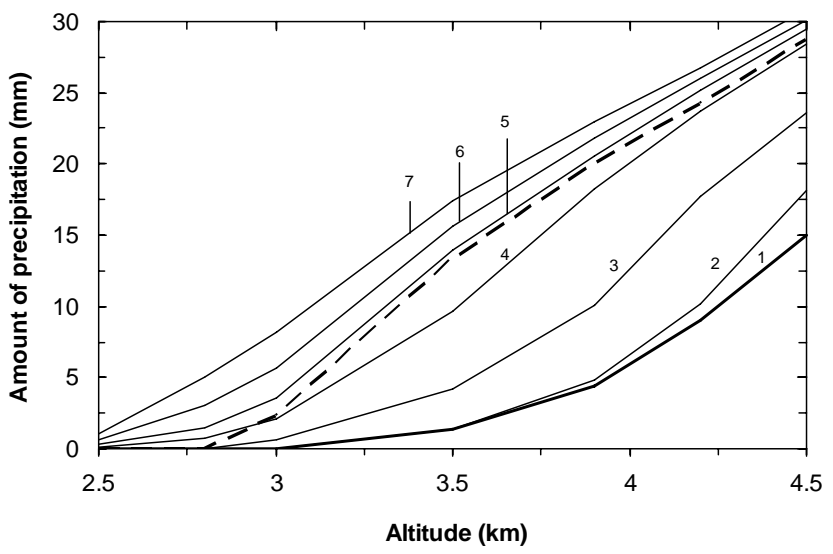

Fig. 10. Simulated total amount of precipitations, defined as $r>200 \mu \mathrm{m}$, from clouds of different thicknesses without the introduction of particles (1), with the $12 \mathrm{~kg} / \mathrm{km}^{2}$ particles of the South African flares consumed (2), with $2.4,6,12,24,48 \mathrm{~kg} / \mathrm{km}^{2}$ of the salt powder (curves 3-7, respectively). Dashed lines refer to the introduction of $64 \mathrm{~kg} / \mathrm{km}^{2}$ of salt particles with the radii of $1 \mu \mathrm{m}$. The precipitation is calculated at the level of cloud base.

$2.4 \mathrm{~kg} / \mathrm{km}^{2}$ of the salt powder the precipitation amounts falling from the clouds with the thicknesses over $4 \mathrm{~km}$ are greater than at cloud modification with the pyrotechnic flares (at the consumption of pyrotechnic particles of $12 \mathrm{~kg} / \mathrm{km}^{2}$ ).

The modification effect obtained in case of a salt powder is also significantly higher than that achieved with larger salt particles with narrow particle size distributions. Fig. 10 gives the calculation results for precipitation at seeding with salt particles with effective radii of $1 \mu \mathrm{m}$ at optimal mass concentration $1.1 \mathrm{mg} / \mathrm{m}^{3}$. The cloud drop spectrum at the initial stage of condensation at seeding by these particles is shown in Fig. 9. The consumption of the agent per $1 \mathrm{~km}^{2}$ of the seeding area for such particles, when they are introduced into the $60-\mathrm{m}$ subcloud layer, makes $64 \mathrm{~kg}$. From Fig. 10 it is seen that at seeding with such particles the modification effect appears almost the same as in case of using the salt powder with its consumption of $12 \mathrm{~kg} / \mathrm{km}^{2}$. It means that the consumption of the salt powder is by 5 times less than that of the salt particles with the radii of $1 \mu \mathrm{m}$.

The calculation results demonstrated that in case of cloud seeding with salt powders at their consumption of $24 \mathrm{~kg} / \mathrm{km}^{2}$, additional precipitation amounts may be obtained from clouds with $2.5<H<3.5 \mathrm{~km}$. Such clouds are not giving significant precipitation under natural conditions. Maximum effect of modification - the greatest precipitation amounts - is realized at the consumption of $48 \mathrm{~kg} / \mathrm{km}^{2}$ of the salt powder (the upper curve in Fig. 10). Here, as the analysis of numerical simulation results shows, very few cloud drops remain in the cloud. Practically, almost all of the cloud water transforms into rain drops and precipitates. Therefore, a further increase in consumption of the agent (over $48 \mathrm{~kg} / \mathrm{km}^{2}$ ) does not lead to a significant additional precipitation enhancement.

\section{Conclusions}

The results of experiments carried out in the cloud chamber at the conditions corresponding to the formation of convective clouds have shown that:

- The introduction of the salt powder before a cloud medium is formed results in the formation on the largedrop "tail" and in the broadening of drop size spectrum. This result is a positive factor for stimulating coagulation processes in clouds and for subsequent formation of precipitation in them.

- No impact is observed on the fine-droplet spectrum fraction formed on background condensation nuclei even at moderate amounts of the powder introduced.

- Seeding with the salt powder leads also to enlargement of the whole population of cloud drops and to a decrease of their total concentration as compared to the background cloud medium. With the introduction of increasing mass concentration of salt particles, this effect increases practically linearly. This factor also leads to a positive modification effect for stimulating the conversion of cloud water into rain drops.

- At the introduction of very high concentration of the powder no "overseeding" is observed, i.e., increase of drop concentration along with reduction of their size is not observed.

The results of numerical simulations of cloud medium formation at the initial stage of condensation have shown that:

- The introduction of the salt powder into the convective subcloud layer leads to the appearance of a large-drop "tail" in the cloud drop size distribution. The shape of the spectrum in the large-drop region is determined by the salt powder particles spectrum.

- The shape of the spectrum of drops formed on background condensation nuclei does not change at rather high concentrations of the powder introduced. This means that the introduction of the salt powder does not change much the conditions of the formation of cloud drops on the background aerosol particles, except for at very high mass concentration. This result is confirmed by the experimental data obtained in the cloud chamber.

- As the analysis of numerical simulation results shows, the transformation of cloud drop spectra induced by the introduction of the salt powder results in much more intense coagulation processes in clouds as compared to the case of cloud modification with particles from hygroscopic flare at the same mass concentration.

- The salt powder results also in much more intense coagulation also with respect to hygroscopic particles having 
1- $\mu \mathrm{m}$ very narrow particle size distribution of the same mass concentration.

The calculated rainfall amounts of the numerical simulations with a 1-dimensional numerical model of a warm convective cloud have shown that:

- The effect of the salt powder on clouds (total amounts of additional rain) is significantly higher than that caused by the use of hygroscopic flares at comparable consumptions of seeding agents (of the order of $10 \mathrm{~kg} / \mathrm{km}^{2}$ ).

- At the consumptions of the salt powders over $20 \mathrm{~kg} / \mathrm{km}^{2}$ rainfall can be obtained from otherwise non-precipitating clouds with thicknesses of $2.5<\mathrm{H}<3.5 \mathrm{~km}$. At the consumptions of about $50 \mathrm{~kg} / \mathrm{km}^{2}$ of the powder the maximum effect of modification - maximum precipitation amounts - is realized. A further increase of the amounts of the salt powder introduced into a cloud does not result in significant additional precipitation amounts. Owing to a fairly simplified description of cloud development in the present 1-dimentional model, the consumptions of the salt powders indicated above should be regarded as estimates. For more accurate calculations it is necessary to use more realistic 2 or 3 -dimensional cloud model with full ice microphysics and dynamic feedbacks to the precipitation forming processes.

In summary, the experimental data and the results of numerical simulations presented here and elsewhere show that a salt powder milled to size of several $\mu \mathrm{m}$ is more effective in initiating warm rain than hygroscopic flares. The calculated amounts of seeding material reach an order of $10 \mathrm{~kg}$ salt per $\mathrm{km}^{2}$ of seeded cloud. The needed mass is even larger for a smaller effect when using hygroscopic flares. This requires seeding amounts of hundreds of $\mathrm{kg}$ per a seeding flight. Dispersion of such quantities is not feasible with hygroscopic flares, but has been demonstrated practical with salt powder (Rosenfeld et al., 2010).

It should be cautioned that faster initiation of warm rain always leads to more rainfall on the ground (e.g., Rosenfeld et al., 2008). Net additional rainfall can be expected as long as cloud tops do not reach the minimum depth for onset of precipitation. In any case, the added rainfall amounts should be investigated in the framework of randomized cloud seeding experiments coupled with detailed model simulations.

Edited by: D. J. Cziczo

\section{References}

Bruintjes, R. T., Breed, D. W., Salazar, V., Dixon, M. J., Kane, T., Foote, G. B., and Brown, B. G.: Overview and results from the Mexican hygroscopic seeding experiment, Preprints, 15th Conf. on Planned and Inadvertent Weather Modification, Amer. Meteor. Soc., 45-48, 2001.

Cooper, W. A., Bruintjes, R. T., and Mather, G. K.: Calculations pertaining to hygroscopic seeding with flares, J. Appl. Meteorol., 36, 1449-1469, 1997.

Drofa, A. S.: Formation of cloud microstructure: the role of hygroscopic particles, Atmos. Ocean. Phys., 42, 326-336, 2006.

Drofa, A. S.: Numerical Simulation of the Action on a Warm Convective Cloud by Hygroscopic Particles, Atmos. Ocean. Phys., 43, 574-585, 2007.

Drofa, A. S.: Studying a Possibility to Stimulate Precipitation from Warm Convective Clouds by Hygroscopic Particles Based on Numerical Simulation Results, Atmos. Ocean. Phys., 44, 435449, 2008.

Drofa, A. S.: Studies of a Modification Effect from a Warm Convective Cloud Seeding by Hygroscopic Particles Based on $\mathrm{Nu}$ merical Simulation Results, Atmos. Ocean. Phys., 46, 328-338, 2010.

Heyder, J. and Gebhart, J.: Optimization of Response Function of Light Scattering Instruments for Size Evolution of Aerosol Particles. Appl. Opt., 18, 734-739, 1979.

Kolomiets, S. M., Meshunenkov, N. I., and Smirnov, V. V.: A Laser Aerosol Analyzer "Delta" for Air Purity Control, Opticomechanical industry, in Russian, 12, 21-24, 1989.

Kuba, N. and Murakami, M.: Effect of Hygroscopic Seeding on Warm Rain Cloud - Numerical Study Using a Hybrid Cloud Microphysical Model, Atmos. Chem. Phys., 10, 3335-3351, doi:10.5194/acp-10-3335-2010, 2010.

Lahav, R. and Rosenfeld, D.: The Search for the Optimal Size of Hygroscopic Seeding Particles, Proceedings of the 16-th Conference on Planned and Inadvertent Weather Modification, San Diego, USA, 2005.

Mather, G. K., D. E. Terblanche, F. E Steffens, and L. Fletcher: Results of the South African Cloud-seeding Experiments Using Hygroscopic Flares, J.Appl. Meteor., 36, 1433-1447, 1997.

Mazin, I. P. and Shmeter, S. M.: Clouds. Structure and Physical formation, in Russian, Leningrad, Gidrometeoizdat, 1983.

Pruppacher, H. R. and Klett, J. D.: Microphysics of Cloud and Precipitation, 2d ed., Oxford University Press, 1997.

Romanov, N. P. and Zhukov, G. P.: Thermodynamic Relations for a Fog Chamber, Russ. Meteorol. Hydrol., 10, 27-37, 2000.

Romanov, N. P.: Calculation of Operating Parameters of $90^{\circ}-\mathrm{De}-$ gree Photoelectric Counters, in Russian, Trudy IEM, Moscow, Gidrometeoizdat, 92-108, 1991.

Rosenfeld, D., Lohmann, U., Raga, G. B., O’Dowd, C. D., Kulmala, M., Fuzzi, S., Reissell, A., and Andreae, M. O.: Flood or Drought: How Do Aerosols Affect Precipitation?, Science, 321, 1309-1313, 2008

Rosenfeld, D., Axisa, D., Woodley, W. L., and Lahav, R.: A Quest for Effective Hygroscopic Cloud Seeding, J. Appl. Meteorol. Clim., 49, 1548-1562, 2010.

Segal, Y., Khain, A., Pinsky, M., and Rosenfeld, D.: Effects of Hygroscopic Seeding on Raindrop Formation as Seen from Simulations Using a 2000-bin Spectral Cloud Parcel Model, Atmos. Res., 71, 3-34, 2004. 
Segal, Y., Pinsky, M., and Khain, A.: The Role of Competition Effect in the Raindrop Formation, Atmos. Res., 83, 106-118, 2007.

Singh, C., Singh, R. N., and Pillai, P. C.: A Study of Geometrical Factor in Optical Particle Counters, Opt. Applicata, 12, 231-242, 1982.

Shmeter, S. M.: Thermodynamics and Physics of the Convective Clouds, in Russian, Leningrad, Gidrometeoizdat, 1987.
Silverman, B. A.: A critical assessment of hygroscopic seeding of convective clouds for rainfall enhancement, Bull. Amer. Meteor. Soc., 84, 1219-1230, 2003.

World Meteorological Organization: Report of the WMO workshop on hygroscopic seeding, WMP Report No. 35, World Meteor. Org., WMO/TD, No. 1006, 2000. 